Homology, Homotopy and Applications, vol.9(1), 2007, pp.467-483

\title{
EQUIVARIANT MORSE RELATIONS
}

\author{
MAHUYA DATTA AND NEETA PANDEY
}

(communicated by Ronald Brown)

\begin{abstract}
For a finite group $G$, Costenoble and Waner defined a cellular (co-)homology theory for $G$-spaces $X$, which is graded on virtual representations of the equivariant fundamental groupoid $\pi_{G}(X)$. Using this homology, we associate an infinite (Morse) series with an equivariant Morse function $f$ defined on a closed Riemannian $G$-manifold $M$. Wasserman has shown that when the critical locus of $f$ is a disjoint union of orbits, $M$ has a canonical decomposition into disc bundles. We show that if this decomposition 'corresponds' to a virtual representation $\gamma$ of $\pi_{G}(M)$, then the Morse relations are satisfied by the ' $\gamma$ th homology groups'. For semi-free $G$-actions, we characterise the Morse functions which naturally give rise to such representations $\gamma$ of $\pi_{G}(M)$. We also show that corresponding to any equivariant Morse function on a $\mathbb{Z}_{2}$-manifold, it is always possible to define virtual representations $\gamma$ so that the Morse relation is satisfied by the ' $\gamma$ th homology groups'. In particular, the Morse relation is satisfied by Bredon homology.
\end{abstract}

\section{Introduction}

Let $G$ be a finite group and $M$ a closed Riemannian $G$-manifold. A natural generalisation of a Morse function in the equivariant context is a smooth $G$-map $f: M \longrightarrow \mathbb{R}$ whose critical locus is a disjoint union of non-degenerate critical orbits. Such functions are dense in the space of all $G$-equivariant smooth real-valued functions on $M[\mathbf{1 0}]$. Morse theory of these functions has been discussed by Wasserman as part of a more general study (see [10]). If $f$ is an equivariant Morse function of the above type on $M$, then $M$ is equivariantly diffeomorphic to $\left(N_{1}, f\right) \cup_{g_{2}}$ $\left(N_{2}, f\right) \cup_{g_{3}} \cdots \cup_{g_{k}}\left(N_{k}, f\right)$, where $\left(N_{i}, f\right)$ are handle-bundles over orbits, that is, the $N_{i}$ are of the form $G \times_{H_{i}}\left(D V_{i} \times D W_{i}\right)$ for some orthogonal $H_{i}$-representation spaces $V_{i}$ and $W_{i}$. These handle-bundles are attached successively along $G \times_{H_{i}}\left(D V_{i} \times S W_{i}\right)$ by the equivariant maps $g_{i}$. Thus $M$ has the equivariant homotopy type of $\left(G \times_{H_{1}} D W_{1}\right) \cup_{g_{2}}\left(G \times_{H_{2}} D W_{2}\right) \cup_{g_{3}} \cdots \cup_{g_{k}}\left(G \times_{H_{k}} D W_{k}\right)([\mathbf{1 0}])$. The disc-bundles $\left(G \times_{H_{i}} D W_{i}\right)$ will be called Morse cells associated to $f$ and the above decomposition will be referred as the Morse complex of $f$.

Received November 22, 2006, revised February 7, 2007; published on April 3, 2007.

2000 Mathematics Subject Classification: 57R70, 57R91, 55N25, 55N91.

Key words and phrases: equivariant Morse function, $R O\left(\pi_{G}(X)\right)$ graded homology, Morse relation.

Copyright (C) 2007, International Press. Permission to copy for private use granted. 
In our earlier paper [5], we tried to establish Morse relations for equivariant Morse functions on $M$ using Bredon cohomology. The Bredon homology and cohomology theories do not support the above type of cells. Therefore, it is not surprising that we can not explain Morse theory completely in terms of Bredon cohomology.

A more general cell structure which includes the disc bundles over orbits as cells has been introduced by Costenoble and Waner $[\mathbf{3}]$. These cell structures on a $G$-space $X$ are parametrised by 'admissible' virtual representations $\gamma$ of the equivariant fundamental groupoid $\pi_{G}(X)$. A representation $\gamma$ of dimension $n$ essentially associates to each $G$-map $x: G / H \longrightarrow X$ a cell of the form $G \times_{H} D W$ for some $n$-dimensional $H$-representation space $W$ so that corresponding to each morphism in $\pi_{G}(X)$ there is a $G$-disc bundle map over the corresponding orbits [2]. A representation $\gamma$ then determines 'admissible' cell structures which are called $G$-CW $(\gamma)$ structures on $X$. This concept can be canonically extended to admissible virtual representations of $\pi_{G}(X)$. If $\gamma$ is the zero-dimensional trivial representation, then the corresponding cell structure consists of Bredon cells. With this new definition of $G$-CW complexes, Costenoble and Waner have defined an equivariant cellular homology and cohomology theory graded on $R O\left(\pi_{G}(X)\right)$, the set of isomorphism classes of virtual representations.

In this paper we try to explain Morse relations for an equivariant Morse function on a Riemannian $G$-manifold in the light of this $R O\left(\pi_{G}(X)\right)$-graded homology theory. After discussing the notion of generalised $G$-CW-complexes and the $R O\left(\pi_{G}(X)\right)$-graded homology and cohomology theory in Sections 2 and 3 respectively, we prove the following in Section 4:

Let $f: M \longrightarrow \mathbb{R}$ be an equivariant Morse function on a Riemannian $G$-manifold $M$ whose critical locus is a disjoint union of $G$-orbits. If the Morse complex determined by $f$ is a $G$-CW $(\gamma)$-complex for some admissible virtual representation $\gamma$ of $\pi_{G}(M)$, where each Morse cell occurs as a cell of the $\gamma$-complex, then the $\gamma$ th homology groups, $H_{\gamma+n}^{G}, n \in \mathbb{Z}$, explain the Morse relations for $f$.

Moreover, when the $G$-action is semi-free, we characterise the Morse functions which naturally give rise to such admissible virtual representations $\gamma$ of $\pi_{G}(M)$.

In Section 5 , we show that if $M$ is a $\mathbb{Z}_{2}$-manifold, then it is always possible to choose an admissible virtual representation $\gamma$ of $\pi_{G}(M)$ so that the Morse complex is a $G-\mathrm{CW}(\gamma)$-complex, each Morse cell being a subcomplex of it. In particular we may choose $\gamma$ to be trivial to get a Bredon cell structure. We also show that the Morse relation is satisfied in this case.

\section{Generalised $G$-CW-complexes}

In this section we shall briefly discuss the basic concept of generalised $G$-CWcomplexes introduced by Costenoble and Waner in $[\mathbf{3}]$.

A cell of type $G / H$ is a space of the form $G \times_{H} D(W)$, where $D(W)$ is the unit disc of an orthogonal $H$-representation space $W$. Thus a cell is canonically associated with an orthogonal $G$-vector bundle over an orbit. Recall that an arbitrary $G$ vector bundle over an orbit $G / H$ is always of the form $G \times_{H} W \longrightarrow G / H$. The dimension of the cell is defined to be the dimension of the representation space $W$. 
The canonical inclusion map $G / H \longrightarrow G \times_{H} D(W)$ is called the centre of the cell.

A cell complex is a $G$-space obtained from a collection of such cells via equivariant attaching. If $X$ is a cell complex, then the centre of a cell of $X$ defines a $G$-map $x: G / H \longrightarrow X$, that is, an element of $\pi_{G}(X)$. Recall that the equivariant fundamental groupoid $\pi_{G}(X)$ is the category of $G$-maps from orbits into $X$. A morphism from $x: G / H \longrightarrow X$ to $y: G / K \longrightarrow X$ in this category is an equivalence class of a pair $(\sigma, \omega)$, where $\sigma: G / H \longrightarrow G / K$ is a $G$-map and $\omega$ is a $G$-homotopy from $x$ to $y \circ \sigma[\mathbf{1}]$.

It follows from the above that each cell complex $X$ determines a map which takes an $x: G / H \longrightarrow X$ (which corresponds to the centre of a cell) onto a vector bundle over $G / H$, which in turn is completely determined by a representation of $H$. This motivates the following:

Definition 2.1. Let $X$ be a $G$-space. An $l$-dimensional representation $\gamma$ of $\pi_{G}(X)$ is a functor that assigns to each element $x: G / H \longrightarrow X$ of the groupoid an orthogonal $G$-bundle over $G / H$ of dimension $l$. Any morphism $(\sigma, \omega): x \rightarrow y$ in $\pi_{G}(X)$ maps onto the homotopy class of a $G$-bundle map $\gamma(\sigma, \omega): \gamma(x) \rightarrow \gamma(y)$, the homotopy being through orthogonal maps, so that equivalent morphisms in $\pi_{G}(X)$ are mapped onto the same homotopy class of $G$-bundle maps $[\mathbf{3}, \mathbf{2}]$.

The representation is orientable if for any two objects $x: G / H \longrightarrow X$ and $y: G / K \longrightarrow X$ of $\pi_{G}(X)$ and any two morphisms $(\sigma, \omega),\left(\sigma, \omega^{\prime}\right): x \rightarrow y$ in $\pi_{G}(X)$, $\gamma(\sigma, \omega)=\gamma\left(\sigma, \omega^{\prime}\right)[\mathbf{2}]$.

A $G$-space $X$ with a representation $\gamma$ of its equivariant fundamental groupoid will be denoted by $(X, \gamma)$.

An $l$-dimensional representation $\gamma$ is said to be trivial if it assigns the trivial bundle $G / H \times \mathbb{R}^{l}$ to each $x: G / H \longrightarrow X$. Also, for any morphism $(\sigma, \omega): x \rightarrow y$ in $\pi_{G}(X), \gamma(\sigma, \omega)=\sigma \times \mathrm{id}_{\mathbb{R}}$.

It is known that a representation is orientable if and only if it has an orientation in the sense that there is a map from it to the universal orientable representation [2]. We will assume that all representations considered here are oriented.

Example 2.2.

(i) If $V$ is an orthogonal representation space of a group $G$, then given any $G$ space $X$ we can define a representation (which we also denote by $V$ by an abuse of notation) that takes an element $x: G / H \longrightarrow X$ in $\pi_{G}(X)$ onto the vector bundle $G / H \times V$.

(ii) If $\xi$ is a vector bundle over $X$, then it defines a representation $\tilde{\xi}$ of $\pi_{G}(X)$ by associating the pullback bundle $x^{*} \xi$ to an element $x: G / H \longrightarrow X$.

Definition 2.3. Let $\gamma$ be a representation of $\pi_{G}(X)$ for some $G$-space $X$. Let $\phi: Y \longrightarrow X$ be a $G$-map. The induced representation $\phi^{*} \gamma$ on $\pi_{G}(Y)$ is defined by $\phi^{*}(\gamma)(x)=\gamma(\phi \circ x)$, where $x \in \pi_{G}(Y)$.

Observation 2.4.

(i) Let $X$ be a $G$-space such that the fixed point set $X^{H}$ is simply connected for each subgroup $H$ of $G$ (for example, if $V$ is a $G$-representation space, then 
the fixed point sets of $V$ are linear subspaces and hence simply connected). Then a representation of $\pi_{G}(X)$ factors through a representation of the orbit category $\mathcal{G}$ of $G$.

(ii) If $V$ is a $G$-representation space, then its tangent bundle $\tau_{V}$ is isomorphic to the product bundle $V \times V$ over $V$. Hence, $\tilde{\tau}_{V}$ is the same as the representation defined by $V$ on $\pi_{G}(V)$ (see Example 2.2(i)).

(iii) If $p: \xi \longrightarrow X$ is a $G$-vector bundle, then $\widetilde{p^{*} \xi}=p^{*} \tilde{\xi}$.

(iv) Consider the $G$-vector bundle $p: G \times_{H} W \longrightarrow G / H$, where $W$ is an orthogonal $H$-representation space. We shall denote this bundle by $\xi_{W}$ in any further reference. The tangent bundle $\tau$ of the total space is isomorphic to $G \times_{H} T W=G \times_{H}(W \times W)=p^{*} \xi_{W}$. Thus it follows from the above observation that the representations $\tilde{\tau}$ and $p^{*} \tilde{\xi}_{W}$ are equivalent.

(v) If a $G$-space $X$ is $G$-homotopy equivalent to a $G$-space $Y$, then their equivariant fundamental groupoids are equivalent. Hence, if $p: \xi \longrightarrow X$ is a $G$ vector bundle, then any representation $\gamma$ of $\pi_{G}(\xi)$ is equivalent to $p^{*} i^{*} \gamma$, where $i: X \longrightarrow \xi$ is the zero section.

In particular, a representation $\gamma$ of $\pi_{G}\left(G \times_{H} D(W)\right)$ is completely determined by $\gamma\left(x_{0}\right)$, where $x_{0}: G / H \longrightarrow G \times_{H} D W$ is the centre of the cell.

Definition 2.5. Let $\gamma$ be a representation of $\pi_{G}(X)$ of dimension $l$. A $G$-CW $(\gamma)$ structure [4] on $X$ consists of a filtration $X^{0} \subset X^{1} \subset \cdots \subset X$ which satisfies the following:

1. The 0-skeleton $X^{0}$ is a disjoint union of orbits $x: G / H \longrightarrow X$ such that $\gamma(x: G / H \longrightarrow X)$ is the trivial bundle $G / H \times \mathbb{R}^{l}$ over $G / H$.

2. The $n$-skeleton $X^{n}=X^{n-1} \cup_{\psi_{n}} \bigcup_{m} e_{m}^{n}$, where $e_{m}^{n}$ are cells of dimension $n$ and $\psi_{n}$ is the attaching map. Let $\phi_{m}^{n}: e_{m}^{n} \longrightarrow X$ denote the characteristic map of $e_{m}^{n}$. Let $x$ be the centre of a $n$-cell $e_{m}^{n}$. If $n \leqslant l, \gamma\left(\phi_{m}^{n} \circ x\right)$ has a codimension $n$ trivial summand and $e_{m}^{n} \equiv D\left(\gamma\left(\phi_{m}^{n} \circ x\right) \ominus \mathbb{R}^{l-n}\right)$. If $n>l, e_{m}^{n} \equiv D\left(\gamma\left(\phi_{m}^{n} \circ\right.\right.$ $\left.x) \oplus \mathbb{R}^{n-l}\right)$.

A $G$-CW $(\gamma)$ structure on $X$ will also be called a $\gamma$ structure on $X$ for brevity. Often, $X$ will be referred as a $G$-CW-complex, suppressing the representation $\gamma$.

Remark 2.6. If $\gamma$ is a trivial representation of any dimension, then $G$-CW $(\gamma)$ structure means the Bredon cell structure. In fact, if $\gamma$ is an arbitrary representation and $\theta$ is a trivial representation, then a $G-\mathrm{CW}(\gamma)$ structure on $X$ is also a $G-\mathrm{CW}(\gamma+\theta)$ structure on $X$ and vice versa.

Remark 2.7. Let $G \times_{H} D(W)$ be a cell of $(X, \gamma)$ and $\phi: G \times_{H} D(W) \longrightarrow X$ be its characteristic map. Then $G \times_{H} W \cong \gamma\left(\phi \circ x_{0}\right) \ominus \mathbb{R}^{k}$ for some $k$, where $x_{0}: G / H$ $\longrightarrow G \times_{H} D(W)$ is the centre of the cell. By Observation 2.4 $(i v), \phi^{*}(\gamma)$ is completely determined by $\phi^{*}(\gamma)\left(x_{0}\right)$ and hence is equivalent to $\tilde{\xi}_{W}$. Explicitly, if $x: G / K \longrightarrow$ $G \times{ }_{H} D W$ is a $G$-map, then $\phi^{*}(\gamma)(x)=\hat{a}^{*} \gamma\left(x_{0}\right)=G \times \times_{a^{-1} K a} W$, where $a \in G$ is such that $x(e K)=a H$. Thus the induced representation of a cell in a $G-C W$ complex is its canonical representation. 
Remark 2.8. If $X$ is a $G$-space and if $\gamma$ is a representation of $\pi_{G}(X)$, then $X$ may not admit a $G$-CW $(\gamma)$ structure; e.g. if $X$ is a point and if $\gamma$ is any non-trivial representation of $\pi_{G}(X)$ then $X$ does not admit a $G$-CW $(\gamma)$-structure. However, it can be proved that given a pair $(X, \gamma)$ there exists a $G$-CW $(\gamma)$-complex $\Gamma X$ such that $(\Gamma X, \gamma) \longrightarrow(X, \gamma)$ is a weak $G$-homotopy equivalence. Here the representation $\gamma$ on $\Gamma X$ means the induced representation on it by the weak homotopy equivalence $[\mathbf{3}, \mathbf{9}]$.

Remark 2.9. Every smooth $G$-manifold $M$ admits a $G$-CW $\left(\tilde{\tau}_{M}\right)$-structure, where $\tau_{M}$ is the tangent bundle of $M[4]$.

Example 2.10. Let $V$ be an orthogonal $G$-representation space and let $S V$ denote the unit sphere in $V$. Let $x \in S V$ and let $H$ be the isotropy subgroup of $x$. The representation space $V$ decomposes into the orthogonal direct sum $V=\langle x\rangle \oplus T_{x}(S V)$ as an $H$-space, where $T_{x}(S V)$ is the tangent space of $S V$ at $x$. Since the $H$ action on $\langle x\rangle$ is trivial, $G \times{ }_{H} V=G \times_{H} T_{x}(S V) \oplus G / H \times \mathbb{R}$. Therefore, by Remark 2.9(ii), $S V$ admits a $G$-CW $(V)$ structure.

Example 2.11. We have already observed that any representation $\gamma$ of $\pi_{G}(G / H)$ is defined by a bundle $p: G \times_{H} W \longrightarrow G / H$ for some $H$-representation space $W$. Hence if $X=G / H$, we may take $\Gamma X=G \times_{H} D W$. Indeed, since $p^{*} \gamma$ is equivalent to the tangent representation on $\pi_{G}(\Gamma X)$ (see Observation $2.4(i v)$ ), it follows by Remark 2.9 that $\Gamma X$ is a $G$-CW $\left(p^{*} \gamma\right)$ complex. The relative structure on $\left(G \times_{H}\right.$ $\left.D W, G \times{ }_{H} S W\right)$ consists of exactly one cell of dimension $\operatorname{dim} W$.

\section{3. $R O\left(\pi_{G} X\right)$-graded homology and cohomology theory}

Let $X$ be a $G$-space and let $R O\left(\pi_{G}(X)\right)$ denote the set of isomorphism classes of virtual representations of $\pi_{G}(X)$. We shall not go into the detailed description of virtual representations here but refer the reader to [3]. If $X$ is compact, then $R O\left(\pi_{G}(X)\right)$ can be characterised as the Grothendieck group of the monoid of the isomorphism classes of representations of $\pi_{G}(X)$.

In this section we shall recall the salient features of the equivariant cellular homology and cohomology theory graded on a subclass of $R O\left(\pi_{G}(X)\right)$, namely the set of isomorphism classes of 'admissible' virtual representations of $\pi_{G}(X)$ following Costenoble and Waner [3].

Let $X$ be a $G$-CW $(\gamma)$-complex. Costenoble and Waner defined a chain complex $C_{*}(X, \gamma)$ where for each $n \geqslant 0, C_{n}(X, \gamma)$ is a $\hat{\pi}_{G}(X)$ group, i.e. a contravariant functor from $\hat{\pi}_{G}(X)$ to $\mathcal{A} b$, where $\hat{\pi}_{G}(X)$ is the associated stable category. The boundary maps are canonically defined natural transformations (see $[\mathbf{3}]$ ).

One way of describing $C_{n}(X, \gamma)$ is $C_{n}(X, \gamma)(x) \cong \sum \hat{\pi} X\left(x, x_{0}\right)$, where the sum runs over the centres $x_{0}$ of the $n$-cells of $X$. Here $\hat{\pi} X\left(x, x_{0}\right)$ denotes the morphisms from $x$ to $x_{0}$ in the stable category.

If $A$ is a $G$-CW $(\gamma)$ subcomplex of $(X, \gamma)$, then the relative chain complex is defined by $C_{*}(X, A, \gamma)=C_{*}(X, \gamma) / C_{*}(A, \gamma)$.

Given a stable local coefficient system (that is, a covariant, additive functor) $T: \hat{\pi}_{G}(X) \longrightarrow \mathcal{A} b$, define abelian groups $C_{n}(X, \gamma ; T)=C_{n}(X, \gamma) \otimes_{\hat{\pi}_{G}(X)} T$, which are obtained by taking the categorical tensor product of the functors $C_{n}(X, \gamma)$ and $T$ 
over $\hat{\pi}_{G}(X)$. The categorical tensor product of two functors as above is obtained by taking the quotient of the usual tensor product of the functors under the equivalence relation

$$
f^{*} a \otimes b \sim a \otimes T(f) b .
$$

Here $f \in \operatorname{Mor}(x, y)$ in $\hat{\pi}_{G}(X), a \in C_{n}(X, \gamma)(y), b \in T(x)$.

For a contravariant functor $T: \hat{\pi}_{G}(X) \longrightarrow \mathcal{A} b$, the cochain complex can be defined as the group of natural transformations $C^{*}(X, \gamma ; T)=\operatorname{Hom}\left(C_{*}(X, \gamma), T\right)$.

Definition 3.1. Let $(X, \gamma)$ be a $G$-CW $(\gamma)$-complex. Define $H_{\gamma+n}^{G}(X ; T)$ as the $(|\gamma|+n)$-dimensional homology groups of $C_{*}(X, \gamma ; T)$ and $H_{G}^{\gamma+n}(X ; T)$ as the $(|\gamma|+n)$-dimensional cohomology groups of $C^{*}(X, \gamma ; T)$ respectively.

The homology and cohomology groups of a $G$-CW $(\gamma)$ pair $(X, A)$ can also be defined in a similar way.

Definition 3.2. If $X$ is a $G$-space with a representation $\gamma$ of its fundamental groupoid, then we define

$$
H_{\gamma+n}^{G}(X ; T):=H_{\gamma+n}^{G}(\Gamma X ; T),
$$

where $(\Gamma X, \gamma)$ is a $G$-CW $(\gamma)$-complex which is weak $G$-homotopy equivalent to $(X, \gamma)$.

Definition 3.3. The reduced homology groups of a based space $X$ with the base point $*$ can be defined by $\tilde{H}_{\gamma+n}(X ; T)=H_{\gamma+n}(X, * ; T)$ for all $n \geqslant 0$.

In general, the above homology and cohomology theories satisfy all the Steenrod axioms except the dimension axiom. If $\gamma=0$, then the resulting homology and cohomology groups reduce to Bredon homology and cohomology groups with twisted coefficient system. Thus the homology and cohomology defined above when evaluated on orbits in integer grading reduce to Bredon homology and cohomology groups of the orbits and hence satisfy the dimension axiom.

Definition 3.4 (Suspension Isomorphism). If $V$ is a $G$-representation space, then for any $G$-CW $(\gamma)$-complex $X$ with a base point there is a natural (suspension) isomorphism

$$
\tilde{H}_{\gamma+n}^{G}(X ; T) \stackrel{\cong}{\longrightarrow} \tilde{H}_{\gamma+V+n}^{G}\left(\Sigma^{V} X ; T\right)
$$

for all $n \geqslant 0$. Here $\Sigma^{V} X$ denotes the smash product of $X$ and the one-point compactification $S^{V}$ of $V[\boldsymbol{7}]$.

More generally, we have an isomorphism

$$
\sigma: H_{\gamma+n}^{G}(X, A ; T) \longrightarrow H_{\gamma+V+n}^{G}((X, A) \times(D V, S V) ; T)
$$

where the product of pairs $(X, A)$ and $(Y, B)$ is defined as $(X \times Y, X \times B \cup A \times Y)$; on the right hand side $\gamma$ should be interpreted as the representation induced from $\gamma$ on $\pi_{G}(X)$ via the projection map $p: X \times Y \longrightarrow X$. In fact, the isomorphism can be proved at the chain level itself.

We have similar isomorphisms in cohomology as well. 
Definition 3.5. For any representation $\gamma$ of $\pi_{G}(X)$ we can define

$$
\tilde{H}_{\gamma-k}^{G}(X ; T):=\tilde{H}_{\gamma}^{G}\left(\Sigma^{k} X ; T\right), \quad \tilde{H}_{G}^{\gamma-k}(X ; T):=\tilde{H}_{G}^{\gamma}\left(\Sigma^{k} X ; T\right),
$$

where $\Sigma^{k} X=X \wedge S^{k}$ is the $k$ th suspension of $X$ for $k \geqslant 0$. This extends the grading to virtual representations of the form $\gamma-\mathbb{R}^{k}$. We shall call such virtual representations 'admissible'.

Let $\delta=\gamma-\mathbb{R}^{k}$ be an admissible virtual representation of $\pi_{G}(X)$. We will say that $X$ is a $G$-CW $(\delta)$-complex if $X$ is a $G$-CW $(\gamma)$-complex (see Remark 2.6).

\section{Morse Relation}

Definition 4.1. Let $\hat{\mathcal{G}}$ be the stable orbit category of $G$ and $\pi: \hat{\pi}_{G}(X) \longrightarrow \hat{\mathcal{G}}$ the canonical functor. Let $T: \hat{\mathcal{G}} \longrightarrow \mathcal{A} b$ be a covariant additive functor such that $T(G / H)=\mathbf{k}$ for all subgroups $H$ of $G$, where $\mathbf{k}$ is a fixed field. All morphisms are field homomorphisms. Consider the stable local coefficient system $T \circ \pi$ on $\hat{\pi}_{G}(X)$ which we denote by $T$ for brevity.

If, for a fixed representation $\gamma$ of $\pi_{G}(X)$, the equivariant homology groups $H_{\gamma+*}^{G}(X ; T)$ are finite dimensional, then we define the formal Poincaré series corresponding to $\gamma$ as

$$
P_{\gamma}^{t}(X ; T)=\sum_{k \in \mathbb{Z}} t^{|\gamma|+k} \operatorname{dim} H_{\gamma+k}^{G}(X ; T)
$$

Morse Series. Let $M$ be a closed Riemannian $G$-manifold, and $f: M \longrightarrow \mathbb{R}$ an equivariant Morse function on $M$. If $N$ is a non-degenerate critical submanifold of $f$ then we can decompose the normal bundle $\nu_{N}$ of $N$ as $\nu_{N}^{+} \oplus \nu_{N}^{-}$, where $\nu_{N}^{+}, \nu_{N}^{-}$ are the subbundles of $\nu_{N}$ on which the Hessian of $f$ is positive and negative definite respectively. The index of $N$ is defined to be the dimension of $\nu_{N}^{-}$.

It can be proved that $M$ is equivariantly diffeomorphic to

$$
\left(D \nu_{N_{0}}^{+} \times D \nu_{N_{0}}^{-}\right) \cup_{g_{1}}\left(D \nu_{N_{1}}^{+} \times D \nu_{N_{1}}^{-}\right) \cup_{g_{2}} \cdots \cup_{g_{r}}\left(D \nu_{N_{r}}^{+} \times D \nu_{N_{r}}^{-}\right),
$$

where $N_{0}, N_{1}, \ldots, N_{r}$ are the critical submanifolds of $f$. The bundles $D \nu_{N_{i}}^{+} \times D \nu_{N_{i}}^{-}$ are attached successively along $D \nu_{N_{i}}^{+} \times S \nu_{N_{i}}^{-}$by the equivariant map $g_{i}[\mathbf{9}]$.

Given a representation $\gamma$ of $\pi_{G}(M)$ we define the Morse series $M_{\gamma}^{t}(f)$ by the formula

$$
M_{\gamma}^{t}(f):=\sum_{i=0}^{r} P_{\gamma_{N_{i}}}^{t}\left(D \nu_{N_{i}}^{-}, S \nu_{N_{i}}^{-} ; T\right)
$$

provided the relevant Poincaré series are defined. Here $\gamma_{N_{i}}$ denotes the representation $\phi_{i}^{*} \gamma$ on $\pi_{G}\left(D \nu_{N_{i}}^{-}\right)$induced by the characteristic map $\phi_{i}: D \nu_{N_{i}}^{-} \longrightarrow M$.

If $N_{i}$ is an orbit of type $G / H_{i}$ then $\nu_{N_{i}}$ is of the form $G \times_{H_{i}}\left(V_{i} \oplus W_{i}\right)$, where $G \times{ }_{H_{i}} W_{i}=\nu_{N_{i}}^{-}$. Hence if $f$ is an equivariant Morse function whose critical locus is the disjoint union of non-degenerate critical orbits, then $M$ has the structure of 
a cell complex. In particular, it has the equivariant homotopy type of

$$
G \times_{H_{0}} D W_{0} \cup_{g_{1}} G \times_{H_{1}} D W_{1} \cup \cdots \cup_{g_{r}} G \times_{H_{r}} D W_{r},
$$

where the cells $G \times_{H_{i}} D W_{i}$ are attached successively along $G \times_{H_{i}} S W_{i}$ by the equivariant maps $g_{i}$, and $\operatorname{dim} W_{i}$ is the index of the critical orbit $G / H_{i} \hookrightarrow X$.

We will refer to (5) as the Morse complex corresponding to the map $f$, and each $G \times_{H_{i}} D W_{i}, 0 \leqslant i \leqslant r$ as a Morse cell associated to $f$.

Observation 4.2. In general, a Morse complex need not be a $G$-CW $(\gamma)$-complex where each Morse cell occurs as a cell of the $\gamma$-complex. For example, consider the $\mathbb{Z}_{2}$-action on $S^{2}$ given by the reflection in the $y z$-plane, and the function $f: S^{2} \longrightarrow \mathbb{R}$ defined by $f(x, y, z)=z$.

If (5) is a $G$-CW-complex then we obtain Morse relations. We first prove the following lemma.

Lemma 4.3. Let $\xi=G \times_{H} W$. Then

$$
H_{\xi+k}^{G}\left(G \times_{H} D W, G \times_{H} S W ; T\right) \cong \begin{cases}T(G / H) & \text { if } k=0 \\ 0 & \text { if } k \neq 0\end{cases}
$$

Proof. Let $(X, A)$ denote the pair $\left(G \times_{H} D W, G \times_{H} S W\right)$ and let $n=\operatorname{dim} W$. Then $X$ is a $G$-CW $(\xi)$-complex and $A$ is a subcomplex of $X$. Moreover, there is exactly one $n$-dimensional cell in the relative complex $(X, A)$. Therefore, for $k<\operatorname{dim} W$, $C_{k}(X, \xi)=C_{k}(A, \xi)$, and for $k>\operatorname{dim} W, C_{k}(X, \xi)$ is zero, as there is no $k$-cell. Hence for $k \neq \operatorname{dim} W, C_{k}(X, A, \xi)=0$. Thus $H_{\xi+k}(X, A)=0$ for all $k \neq 0$. Further note that $C_{n}(A, \xi)=0$ and $C_{n}(X, \xi)(x) \cong \hat{\pi} X\left(x, x_{0}\right)$, where $x_{0}$ denotes the centre of the $n$-cell of $X$. Thus

$$
H_{\xi}(X, A ; T)=C_{n}(X, \xi) \otimes_{\hat{\pi} X} T=\sum_{x_{K}} \hat{\pi} X\left(x_{K}, x_{0}\right) \otimes T\left(x_{K}\right) / \sim \cong T\left(x_{0}\right) .
$$

Here $x_{K}$ denotes the centre of a cell of $X$ of the form $G \times_{K} D V$. The isomorphism is defined on the generators by $F: f \otimes a \mapsto T(f)(a)$ and then extended canonically. First note that $F$ is well-defined. Let $f \in \hat{\pi} X\left(x_{K}, x_{0}\right)$ and $h \in \hat{\pi} X\left(x_{L}, x_{K}\right)$. Then $F(f \otimes T(h)(b))=T(f)(T(h)(b))=T(f h)(b)=T\left(h^{*} f\right)(b)=F\left(h^{*} f \otimes b\right)$.

$F$ is onto because for any $a \in T\left(x_{0}\right), F($ id $\otimes a)=T($ id $) \otimes a=a$. To show that $F$ is one-one, take $f \otimes a \in \hat{\pi} X\left(x_{K}, x_{0}\right) \otimes T\left(x_{K}\right)$ and $g \otimes b \in \hat{\pi} X\left(x_{L}, x_{0}\right) \otimes T\left(x_{L}\right)$ and suppose that $T(f)(a)=T(g)(b)$. Then id $\otimes T(f)(a)=\mathrm{id} \otimes T(g)(b)$ which is the same as $f \otimes a=g \otimes b$ under the equivalence relation.

Theorem 4.4. Let $f: M \longrightarrow \mathbb{R}$ be an equivariant Morse function whose critical locus is the disjoint union of non-degenerate critical orbits $N_{0}, N_{1}, \ldots, N_{r}$ having indices $\lambda_{0}, \lambda_{1}, \ldots, \lambda_{r}$ respectively. Suppose that the Morse complex (5) defined by $f$ is a $G-C W(\gamma)$-complex for some zero-dimensional virtual representation $\gamma$ of 
$\pi_{G}(M)$. Then

$$
\operatorname{dim} H_{\gamma+k}^{G}(M ; T) \leqslant n_{k},
$$

where $n_{k}$ denotes the number of critical orbits of index $k$. Further,

$$
\begin{aligned}
M_{\gamma}^{t}(f) & =\sum_{i=0}^{r} t^{\lambda_{i}}, \\
M_{\gamma}^{t}(f)-P_{\gamma}^{t}(M) & =(1+t) Q(t)
\end{aligned}
$$

for some polynomial $Q(t)$ with positive coefficients.

Proof. Let $M^{a}$ denote the half space $\{x \in M \mid f(x) \leqslant a\}$ in $M$ for $a \in \mathbb{R}$. If $a_{1}<$ $a_{2}<\cdots<a_{n}$ are the critical values of the function $f$, then $M^{a_{j}}$ is obtained from $M^{a_{j-1}}$ by attaching finitely many handle bundles, one handle bundle for each critical orbit. Moreover, these handles are attached disjointly to $M^{a_{j-1}}$. Let $N_{i}, i=$ $m_{j-1}+1, \ldots, m_{j}$ denote the critical orbits of $f$ corresponding to the critical value $a_{j}$ in $f^{-1}\left(a_{j-1}, a_{j}\right]$. Since each $N_{i}$ is an orbit and $\gamma$ is zero-dimensional, we have $\gamma_{N_{i}}+\mathbb{R}^{\lambda_{i}}=p^{*} \tilde{\nu}_{N_{i}}^{-}$for $i=0,1, \ldots, r$ (see Remark 2.7). For brevity, we denote the representation $p^{*} \tilde{\nu}_{N_{i}}^{-}$of $\pi_{G}\left(\nu_{N_{i}}^{-}\right)$by $\nu_{N_{i}}^{-}$. Thus we get

$$
\begin{aligned}
H_{\gamma+k}^{G}\left(M^{a_{j}}, M^{a_{j-1}} ; T\right) & =\oplus_{i=m_{j-1}+1}^{m_{j}} H_{\gamma+k}^{G}\left(D \nu_{N_{i}}^{-}, S \nu_{N_{i}}^{-} ; T\right) \\
& =\oplus_{i=m_{j-1}+1}^{m_{j}} H_{\nu_{N_{i}}^{-}-\lambda_{i}+k}^{G}\left(D \nu_{N_{i}}^{-}, S \nu_{N_{i}}^{-} ; T\right) .
\end{aligned}
$$

Since $N_{i}$ is an orbit, the homology group $H_{\nu_{N_{i}}^{-}-\lambda_{i}+k}^{G}\left(N_{i} ; T\right)$ is nontrivial if and only if $\lambda_{i}=k$ (Lemma 4.3). Now the first inequality follows from the subadditivity of $\operatorname{dim} H_{\gamma+*}^{G}(-; T)[8]$, namely

$$
\operatorname{dim} H_{\gamma+k}^{G}(M ; T) \leqslant \sum_{j=1}^{n} \operatorname{dim} H_{\gamma+k}^{G}\left(M^{a_{j}}, M^{a_{j-1}} ; T\right) .
$$

It is clear from Lemma 4.3 that $M_{\gamma}^{t}(f)$ is defined in this case, and

$$
\begin{aligned}
M_{\gamma}^{t}(f) & =\sum_{i=0}^{r} P_{\nu_{N_{i}}^{-}-\lambda_{i}}^{t}\left(D \nu_{N_{i}}^{-}, S \nu_{N_{i}}^{-} ; T\right) \\
& =\sum_{i=0}^{r} \sum_{k} t^{k} \operatorname{dim} H_{\nu_{N_{i}}^{-}-\lambda_{i}+k}^{G}\left(D \nu_{N_{i}}^{-}, S \nu_{N_{i}}^{-} ; T\right) \\
& =\sum_{i=0}^{r} t^{\lambda_{i}} \quad(\text { by Lemma 4.3). }
\end{aligned}
$$

To prove the last statement, consider a $G$-CW $(\gamma)$-complex $X$ and a subcomplex $Y$ of $X$ such that $X=Y \cup_{\phi} e$, where $e=G \times_{H} D W$ and $\gamma$ is a virtual representation of $\pi_{G}(X)$ of dimension zero so that $\left.\gamma\right|_{e}+\mathbb{R}^{\lambda}=\xi_{W}, \lambda$ being the dimension of $W$. Then $H_{\gamma+k}(X, Y ; T) \cong H_{\xi_{W}-\lambda+k}\left(G \times_{H} D W, G \times_{H} S W ; T\right) \cong T(G / H) \cong \mathbf{k}$ when $k=\lambda$ and zero otherwise. Let us denote the generator of $H_{\gamma}(X, Y ; T)$ by $[e]$. 
It follows from the homology long exact sequence of $(X, Y)$,

$$
\begin{aligned}
0 \rightarrow H_{\gamma+\lambda}(Y ; T) \stackrel{i_{0}}{\longrightarrow} H_{\gamma+\lambda}(X ; T) \stackrel{j_{*}}{\longrightarrow} H_{\gamma+\lambda}(X, Y ; T) \stackrel{\partial}{\longrightarrow} \\
H_{\gamma+\lambda-1}(Y ; T) \stackrel{i_{1}}{\longrightarrow} H_{\gamma+\lambda-1}(X ; T) \rightarrow 0,
\end{aligned}
$$

that if $\partial[e]=0$, then

$$
\operatorname{dim} H_{\gamma+\lambda}(X ; T)=\operatorname{dim} H_{\gamma+\lambda}(Y ; T)+1
$$

and

$$
\operatorname{dim} H_{\gamma+\lambda-1}(X ; T)=\operatorname{dim} H_{\gamma+\lambda-1}(Y ; T) .
$$

On the other hand, if $\partial[e] \neq 0$, then it follows that

$$
\operatorname{dim} H_{\gamma+\lambda}(X ; T)=\operatorname{dim} H_{\gamma+\lambda}(Y ; T)
$$

and

$$
\operatorname{dim} H_{\gamma+\lambda-1}(X ; T)=\operatorname{dim} H_{\gamma+\lambda-1}(Y ; T)-1 .
$$

Therefore attaching a cell amounts to an increment of $t^{\lambda}$ or $-t^{\lambda-1}$ in the Poincaré polynomial. On the other hand, attaching a cell of dimension $\lambda$ contributes $t^{\lambda}$ to the Morse polynomial.

Since $M$ is obtained by successive attachment of cells it follows from the above discussion that $M_{\gamma}^{t}(f)-P_{\gamma}^{t}(M)=(1+t) Q(t)$ for some polynomial $Q(t)$ with positive coefficients.

In the rest of the section we restrict ourselves to semi-free $G$-action on $M$ and characterise Morse functions which satisfy the hypothesis of Theorem 4.4.

Note that a representation $\gamma$ of $\pi_{G}(M)$ determines a unique (up to equivalence) $G$-representation space for each component of the $G$-fixed point set of $M$. When the $G$-action is semi-free, the converse is also true. We have the following result.

Theorem 4.5. Let $G$ act semi-freely on a smooth manifold $M$ and let $f: M \longrightarrow \mathbb{R}$ be an equivariant Morse function whose critical locus is a disjoint union of orbits. $A$ necessary and sufficient condition for (5) to represent a $G$-CW $(\gamma)$-complex for some representation $\gamma$ is the following:

If $p$ is a G-fixed critical point of $f$, then the isomorphism class of $\nu_{p}^{-} \ominus\left(\nu_{p}^{-}\right)^{G}$ depends only on the path component of $p$ in $M^{G}$.

Proof. There are only two orbit types in this case. We define a zero-dimensional orientable virtual representation $\gamma$ of $\pi_{G}(M)$ as follows: Let us choose a fixed critical point $p_{C}$ in $C$ for each component $C$ of $M^{G}$ containing a critical point of $f$. If $x: G \longrightarrow M$ is a $G$-map, define $\gamma(x)=G$. Any morphism between $x, x \circ g: G \longrightarrow M$ is mapped to the bundle map induced by $g$. On the other hand, if $x: G / G \longrightarrow M^{G}$, then there are two cases. If $x$ maps into a component $C$ of $M^{G}$ containing a critical point of $f$, then define $\gamma(G / G \longrightarrow C)=\nu_{p_{C}}^{-} \ominus \mathbb{R}^{\lambda_{p_{C}}}$, where $\lambda_{p_{C}}$ is the index of $f$ at $p_{C}$. Any morphism between $x, y: G / G \longrightarrow C$ is mapped to the identity virtual bundle map on $\nu_{p_{C}}^{-} \ominus \mathbb{R}^{\lambda_{p_{C}}}$. Any morphism between $x: G \longrightarrow M$ and $y: G / G \longrightarrow$ $C$, where $C$ is a component of $M^{G}$ containing a critical point of $f$, maps to the 
bundle map induced by the action $G \times \mathbb{R}^{\lambda_{p_{C}}} \longrightarrow \nu_{p_{C}}^{-}$. If $x$ maps into a component $C$ that does not contain any critical point of $f$, then define $\gamma(G / G \longrightarrow C)=0$. The effect of $\gamma$ on the rest of the morphisms is obvious. Thus $\gamma$ is an admissible orientable virtual representation of $\pi_{G}(M)$ and (5) is a $G$-CW $(\gamma)$-complex.

The necessity of the condition follows from the functorial property of $\gamma$.

Observation 4.6. The hypothesis of Theorem 4.5 is satisfied in the following cases:

1. $G$ is the trivial group so that $\nu_{p}^{-}=\left(\nu_{p}^{-}\right)^{G}$.

2. The $G$-action is free.

3. The $G$-action is semi-free and $G$-fixed set $M^{G}$ is discrete.

Corollary 4.7. Let $G=\mathbb{Z}_{2}$ and let $f: M \longrightarrow \mathbb{R}$ be as in Theorem 4.5. Let $\lambda_{p}$ denote the index of $f$ at a $G$-fixed critical point $p$ and let $\lambda_{p}^{G}$ denote the dimension of $\left(\nu_{p}^{-}\right)^{G}$. If the value of $\lambda_{p}-\lambda_{p}^{G}$ depends only on the path component of $p$ in $M^{G}$, then there exists a representation $\gamma$ of $\pi_{G}(M)$ such that the cell complex (5) defined by $f$ is a $G$-CW $(\gamma)$-complex in which the Morse cells appear as cells of the complex.

Proof. Every action of $G=\mathbb{Z}_{2}$ is semi-free. The proof now follows from Theorem 4.5 with the observation that there are only two irreducible representations of $G$, namely the trivial representation and the antipodal representation.

We now give an example of functions which satisfy the hypothesis of the above corollary.

Example 4.8. Consider the manifold $M=S^{n-1}$ and the function $f: \mathbb{R}^{n} \longrightarrow \mathbb{R}$ defined by $f\left(x_{1}, x_{2}, \ldots, x_{n}\right)=a_{1} x_{1}^{2}+\cdots+a_{n} x_{n}^{2}$, where $0<a_{1}<a_{2}<\cdots<a_{n}$. The restriction of $f$ to $S^{n-1}$ is a Morse function.

Let $e_{j}$ denote the unit vector in $\mathbb{R}^{n}$ having the $j$ th coordinate equal to 1 . For each $j=1, \ldots, n, f$ has two critical points, namely $e_{j}$ and $-e_{j}$, which are both non-degenerate (since $a_{i} \neq a_{j}$ for $i \neq j$ ) and $f$ has the same index $j-1$ at these two points. The number $j-1$ corresponds to the number of $a_{i}$ 's which are strictly less than $a_{j}$.

Let $G=\mathbb{Z}_{2}$ act orthogonally on $S^{n-1}$ so that the fixed point set is the intersection of $S^{n-1}$ with the subspace spanned by $e_{j}, e_{j+1}, \ldots, e_{k}$ for some integers $j, k>j$. Then for the critical point $e_{i}, j \leqslant i \leqslant k, \lambda_{e_{i}}$ is equal to the dimension of the subspace $\left\langle e_{1}, \ldots, e_{i-1}\right\rangle$ while $\lambda_{e_{i}}^{G}=\operatorname{dim}\left\langle e_{j}, \ldots, e_{i-1}\right\rangle$. Thus for any critical point $p$ in $\left(S^{n-1}\right)^{\mathbb{Z}_{2}}, \lambda_{p}-\lambda_{p}^{G}=j-1$ and hence the hypothesis of the above corollary is satisfied.

\section{5. $\quad$ Morse theory on a $\mathbb{Z}_{2}$-manifold}

In the previous section we proved that an equivariant Morse function $f: M \longrightarrow$ $\mathbb{R}$ whose critical locus is a disjoint union of orbits satisfies the Morse relations (Theorem 4.4), provided the Morse complex associated to $f$ gives rise to a $G$-CWcomplex in which each Morse cell appears as a cell of the complex. In general, this condition is not satisfied. 
In this section, restricting ourselves to a $\mathbb{Z}_{2}$-manifold, we canonically define a virtual representation $\gamma$ of $\pi_{G}(M)$ associated to a Morse function $f$. It turns out that the Morse complex determined by $f$ is a $G$-CW $(\gamma)$-complex where each cell of the Morse complex appears as a subcomplex. Next, we fix a coefficient system $T$ and prove Morse relations with homology groups $H_{\gamma+*}(M ; T)$. We also observe that the Morse relations are satisfied by Bredon homology.

Throughout this section $G=\mathbb{Z}_{2}$. Let $\rho_{n}$ denote the antipodal action of $G$ on $\mathbb{R}^{n}$, as well as the corresponding representation of $\mathbb{Z}_{2}$ on $\mathbb{R}^{n}$. Let $\theta_{n}$ be the trivial representation of dimension $n$. The only linear representations of $\mathbb{Z}_{2}$ are of the form $\rho_{k} \oplus \theta_{m}$.

We shall first describe a class of admissible $G$-CW structures on the generalised cells when $G=\mathbb{Z}_{2}$.

Example 5.1. It is clear from the definition of a $G$-CW-complex that there can not be any $\rho_{k}$ structure on $X=D\left(\rho_{l} \oplus \theta_{m}\right)$ for $k>l$. However for $k \leqslant l$ there is a $\rho_{k}$-structure on $X$; we give an explicit description of such a structure.

- First of all, for $0 \leqslant p<k$, we attach successively one $p$-cell $e_{p}$ of type $G / e$ which gives a $\rho_{k}$-structure on $S\left(\rho_{k}\right)$.

- Then for $k \leqslant p \leqslant k+m-1$, we attach two $p$-cells, $e_{p}, \bar{e}_{p}$ of type $G / G$ which gives a $\rho_{k}$-structure on $S\left(\rho_{k} \oplus \theta_{m}\right)$.

- Next, we attach a $(k+m)$-cell $e_{k+m}^{\prime}$ of type $G / G$ whose interior is mapped homeomorphically into the interior of the disc $X$ to obtain a $\rho_{k}$-structure on $D\left(\rho_{k} \oplus \theta_{m}\right)$.

- Finally, for each $p, k+m \leqslant p<m+l$, we attach first a $p$-cell $e_{p}$ of type $G / e$ which lies on the boundary and then a $(p+1)$-cell $e_{p+1}^{\prime}$ of type $G / e$ whose interior is mapped into the interior of $X$.

Definition 5.2 (Definition of $\gamma$ ). Let $M$ be a $G$-manifold and $f: M \longrightarrow \mathbb{R}$ a Morse function whose critical locus is a disjoint union of orbits. Let $C$ be a component of $M^{G}$ containing critical points of $f$. There can be only finitely many critical points in a component. Let $\lambda_{C}=\min \left\{\lambda_{p}-\lambda_{p}^{G}: p\right.$ is a critical point in $\left.C\right\}$. There is at least one critical point $p_{C}$ in $C$ for which $\lambda_{p_{C}}-\lambda_{p_{C}}^{G}=\lambda_{C}$. Further we observe that the bundle $\nu_{p_{C}}^{-} \ominus\left(\nu_{p_{C}}^{-}\right)^{G}$ is isomorphic with $\rho_{\lambda_{C}}$.

If $x: G \longrightarrow M$ is a $G$-map, define $\gamma(x)=G$. On the other hand, if $x: G / G \longrightarrow$ $M^{G}$, then there are two cases. If $x$ maps into a component $C$ of $M^{G}$ containing a critical point $p$ of $f$, then define $\gamma(G / G \longrightarrow C)=\rho_{\lambda_{C}} \ominus \mathbb{R}^{\lambda_{C}}$. If $x$ maps into a component $C$ that does not contain any critical point of $f$, then define $\gamma(G / G \longrightarrow$ $C)=0$. Then $\gamma$ is an admissible virtual representation of $\pi_{G}(M)$, because if $p$ is a $G$-fixed critical point of $f$, then by definition the isomorphism class of $\gamma(p: G / G \longrightarrow$ $M$ ) depends only on the path component of $p$ in $M^{G}$. It is worth noting that the restriction of $\gamma$ to the Morse cell corresponding to a critical point $p$ is $\rho_{\lambda_{C}} \ominus \mathbb{R}^{\lambda_{C}}$, where $C$ is the component of $M^{G}$ containing $p$. As explained in the above example, the Morse cell $D\left(\nu_{p}^{-}\right)$admits a $\rho_{\lambda_{C}}$ structure since $\lambda_{C} \leqslant \lambda_{p}$. Consequently, the Morse complex admits a $G$-CW $(\gamma)$ structure with each Morse cell as a subcomplex. 
Remark 5.3. It is possible to define other $G$-CW $(\gamma)$ structures on the Morse complex so that each Morse cell is a subcomplex. This can be simply done by choosing each $\lambda_{C}$ less or equal to $\min \left\{\lambda_{p}-\lambda_{p}^{G}: p\right.$ is a critical point in $\left.C\right\}$. In particular, we may take $\lambda_{C}=0$ for all $C$ to get a Bredon cell structure. In the subsequent discussion, $\gamma$ will be any of these virtual representations.

Definition 5.4 (Coefficient System $\boldsymbol{T}$ ). Let $G=\mathbb{Z}_{2}$. We define a coefficient system on $\hat{\mathcal{G}}$, the stable orbit category of $G$ whose objects are $G / e$ and $G / G[\mathbf{3}]$. In the following, the arrows will denote the morphisms in the orbit category $\mathcal{G}$.

$\hat{\mathcal{G}}(G / e, G / e)$ is generated by the equivalence classes of the morphisms

$$
\phi_{g}: G \leftarrow G \rightarrow G, g \in G,
$$

where the right arrow is the identity morphism and the left arrow is $\hat{g}$.

$\hat{\mathcal{G}}(G / e, G / G)$ is generated by the equivalence class of the morphism

$$
\alpha: G / e \leftarrow G / e \rightarrow G / G,
$$

where the morphism $G / e \rightarrow G / e$ is the identity map.

$\hat{\mathcal{G}}(G / G, G / e)$ is generated by the equivalence class of the morphism

$$
\bar{\alpha}: G / G \leftarrow G / e \rightarrow G / e,
$$

where $\bar{\alpha}$ is the dual of $\alpha$.

$\hat{\mathcal{G}}(G / G, G / G)$ is generated by the equivalence class of the morphism

$$
\psi: G / G \leftarrow G / e \rightarrow G / G
$$

and the class of the identity isomorphism.

The morphisms satisfy the following composition relations:
(a) $\phi_{g} \phi_{h}=\phi_{g+h}$,
(b) $\phi_{g} \bar{\alpha}=\bar{\alpha}$,
(c) $\alpha \phi_{g}=\alpha$,
(d) $\psi \psi=\oplus_{g \in G} \psi$,
(e) $\bar{\alpha} \alpha=\oplus_{g \in G} \phi_{g}$,
(f) $\alpha \bar{\alpha}=\psi$,
(g) $\psi \alpha=\oplus_{g \in G} \alpha$,
(h) $\bar{\alpha} \psi=\oplus_{g \in G} \bar{\alpha}$.

We fix a coefficient system $T$ which assigns to both $G / e$ and $G / G$ the field $\mathbb{Z}_{2}$. Further, $T\left(\phi_{g}\right)=1$ for all $g \in G$ (so that $T(G / e)$ is a trivial $G$-module), $T(\alpha)=0$, $T(\bar{\alpha})=0$ and $T(\psi)=0$.

Proposition 5.5. For any pair of $G$-CW-complexes $(X, Y)$,

$$
H_{*}^{G}(X, Y ; T) \cong H_{*}\left(X / G, Y / G \cup X^{G} ; \mathbb{Z}_{2}\right) \oplus H_{*}\left(X^{G}, Y^{G} ; \mathbb{Z}_{2}\right) .
$$

Proof. First observe that the coefficient system $T$ defined as above is naturally equivalent to the direct sum of two coefficient systems. Let $T_{1}$ be the coefficient system such that $T_{1}(G / e)=\mathbb{Z}_{2}, T_{1}(G / G)=0, T_{1}\left(\phi_{g}\right)=1$ for all $g \in G$ and $T_{1}$ maps all other morphisms to 0 . Let $T_{2}$ be the coefficient system such that $T_{2}(G / e)=0$, $T_{2}(G / G)=\mathbb{Z}_{2}$, and $T_{2}$ maps all non identity morphisms to 0 . Then $T$ is naturally equivalent to the direct sum of $T_{1}$ and $T_{2}$. 
This implies that

$$
H_{*}^{G}(X, Y ; T) \cong H_{*}^{G}\left(X, Y ; T_{1}\right) \oplus H_{*}^{G}\left(X, Y ; T_{2}\right) .
$$

On the other hand, $H_{*}\left(X / G, Y / G \cup X^{G} ; \mathbb{Z}_{2}\right)$ defines an integer graded ordinary equivariant homology theory on pairs $(X, Y)$. Its value on orbits is the same as the value of $H_{*}^{G}\left(-; T_{1}\right)$ on orbits. By the uniqueness of Bredon homology it follows that $H_{*}^{G}\left(X, Y ; T_{1}\right) \cong H_{*}\left(X / G, Y / G \cup X^{G} ; \mathbb{Z}_{2}\right)$. A similar argument shows that $H_{*}^{G}\left(X, Y ; T_{2}\right) \cong H_{*}\left(X^{G}, Y^{G} ; \mathbb{Z}_{2}\right)$.

Lemma 5.6. With the coefficient system $T$ as above,

$$
H_{i}^{G}\left(D\left(\rho_{l} \oplus \theta_{m}\right), S\left(\rho_{l} \oplus \theta_{m}\right) ; T\right) \cong \begin{cases}\mathbb{Z}_{2} & \text { for all } m \leqslant i \leqslant l+m \\ 0 & \text { otherwise. }\end{cases}
$$

We shall give a proof of this lemma in the next section.

Corollary 5.1. The $\rho_{k}$-homology of $\left(D\left(\rho_{l} \oplus \theta_{m}\right), S\left(\rho_{l} \oplus \theta_{m}\right)\right)$, for $0 \leqslant k \leqslant l$, is given as

$$
H_{\rho_{k}+i}^{G}\left(D\left(\rho_{l} \oplus \theta_{m}\right), S\left(\rho_{l} \oplus \theta_{m}\right) ; T\right) \cong \mathbb{Z}_{2}, \quad \text { for all } m \leqslant i \leqslant l+m-k,
$$

and zero otherwise. Hence the corresponding Poincaré polynomial is

$$
P_{\rho_{k}}^{t}\left(D\left(\rho_{l} \oplus \theta_{m}\right), S\left(\rho_{l} \oplus \theta_{m}\right) ; T\right)=\sum_{i=k+m}^{l+m} t^{i} .
$$

Proof. It is enough to note that by the Suspension Theorem,

$$
\begin{aligned}
H_{\rho_{k}+i}^{G}\left(D\left(\rho_{l} \oplus \theta_{m}\right), S\left(\rho_{l} \oplus \theta_{m}\right) ; T\right) & =H_{\rho_{k}+i}^{G}\left(\Sigma^{\rho_{k}} D\left(\rho_{l-k} \oplus \theta_{m}\right), \Sigma^{\rho_{k}} S\left(\rho_{l-k} \oplus \theta_{m}\right) ; T\right) \\
& \cong H_{i}^{G}\left(D\left(\rho_{l-k} \oplus \theta_{m}\right), S\left(\rho_{l-k} \oplus \theta_{m}\right) ; T\right) .
\end{aligned}
$$

The result then follows from the above lemma.

Theorem 5.7. Let $M$ be a $\mathbb{Z}_{2}$-manifold and $f: M \longrightarrow \mathbb{R}$ an equivariant Morse function whose critical locus is a disjoint union of orbits. Let $T$ and $\gamma$ be as defined above. Then $f$ satisfies the Morse relation $M_{\gamma}^{t}(f)-P_{\gamma}^{t}(f)=(1+t) Q(t)$, where $Q(t)$ is a polynomial with positive coefficients.

In particular, the Morse relations are satisfied in Bredon homology.

Proof. Let $D\left(\rho_{l} \oplus \theta_{m}\right)$ be attached as $f$ passes through a critical point $p$, and let $\gamma$ restrict to $\rho_{k} \ominus \mathbb{R}^{k}$ on $D\left(\rho_{l} \oplus \theta_{m}\right)$ where $k \leqslant l$. We have seen, in Example 5.1, that the relative $\rho_{k}$-structure on $\left(D\left(\rho_{l} \oplus \theta_{m}\right), S\left(\rho_{l} \oplus \theta_{m}\right)\right)$ is given by one $(m+k)$-cell $e_{m+k}$ of type $G / G$ and one $i$-cell $e_{i}$ of type $G / e$ for all $m+k+1 \leqslant i \leqslant l+m$.

Suppose the cells are attached one by one in the order of increasing dimension. As we noted in the proof of Theorem 4.4, attaching a cell of dimension $n$ causes an increment of $t^{n}$ or $-t^{n-1}$ in the Poincaré polynomial. Thus for each $n, m+k \leqslant$ $n \leqslant m+l$, attachment of the cell $e_{n}$ contributes an increment of $a t^{n}-b t^{n-1}$ in the Poincaré polynomial, where $(a, b)$ is either $(1,0)$ or $(0,1)$. Summing over all $n$ between $m+k$ and $m+l$, we get the value of $\Delta_{p}\left(P_{\gamma}^{t}\right)$, the increment in the 
Poincaré polynomial while $f$ passes through the critical point $p$. On the other hand, it follows from the above corollary that the contribution to Morse polynomial is $\sum_{n=m+k}^{m+l} t^{n}$ for the same critical point $p$. Therefore $\Delta_{p}\left(M_{\gamma}^{t}(f)-P_{\gamma}^{t}\right)=(1+t) Q(t)$ for some polynomial $Q(t)$.

\section{Proof of Lemma 5.6}

Proof of Lemma 5.6. Let us denote $S\left(\rho_{l} \oplus \theta_{r}\right)$ by $X_{r}$ for all $r \geqslant 0$. Then $D\left(\rho_{l} \oplus \theta_{m}\right)$ is a cone over $X_{r}$ and we denote it by $C X_{r}$. Thus we need to compute the relative homology groups of $\left(C X_{r}, X_{r}\right)$.

Let $r \geqslant 0$. The cone $C X_{r}$ over $X_{r}$ is contractible. Hence by the Bredon homology long exact sequence of $\left(C X_{r}, X_{r}\right)$, we get

$$
H_{i}^{G}\left(C X_{r}, X_{r} ; T\right) \cong H_{i-1}^{G}\left(X_{r} ; T\right) \text { for all } i \geqslant 2 .
$$

Thus we need to compute the homology groups $H_{0}^{G}\left(C X_{r}, X_{r} ; T\right), H_{1}^{G}\left(C X_{r}, X_{r} ; T\right)$ and $H_{i}^{G}\left(X_{r} ; T\right)$ for $i \geqslant 1$.

First, observe that $\left(C X_{r}\right)^{G}=D^{r}$ and $\left(X_{r}\right)^{G}=S^{r-1}$, while $X_{0}=\emptyset$ by convention. By using Proposition 5.5 we obtain

$$
\begin{aligned}
& H_{0}^{G}\left(C X_{r} ; T\right) \cong \begin{array}{ll}
0 \oplus \mathbb{Z}_{2} & \text { for all } r \geqslant 0
\end{array} \\
& H_{0}^{G}\left(X_{r} ; T\right) \cong \begin{cases}\mathbb{Z}_{2} \oplus 0 & \text { if } r=0 \\
0 \oplus\left(\mathbb{Z}_{2} \oplus \mathbb{Z}_{2}\right) & \text { if } r=1 \\
0 \oplus \mathbb{Z}_{2} & \text { if } r \geqslant 2\end{cases} \\
& H_{0}^{G}\left(C X_{r}, X_{r} ; T\right) \cong \begin{cases}0 & \text { if } r \geqslant 1 \\
0 \oplus \mathbb{Z}_{2} & \text { if } r=0 .\end{cases}
\end{aligned}
$$

We write the homology in split form to indicate that the components correspond to the splitting mentioned in Proposition 5.5.

Next, considering the exact sequence

$$
0 \rightarrow H_{1}^{G}\left(C X_{r}, X_{r} ; T\right) \rightarrow H_{0}^{G}\left(X_{r} ; T\right) \stackrel{\phi}{\rightarrow} H_{0}^{G}\left(C X_{r} ; T\right) \rightarrow H_{0}^{G}\left(C X_{r}, X_{r} ; T\right) \rightarrow 0,
$$

we obtain that

$$
H_{1}^{G}\left(C X_{r}, X_{r} ; T\right) \cong \begin{cases}\mathbb{Z}_{2} & \text { if } r=0,1 \\ 0 & \text { if } r \geqslant 2 .\end{cases}
$$

It now remains to determine the homology groups of $X_{r}$. Since the suspension $\Sigma X_{r}$ of $X_{r}$ is $X_{r+1}$ for all $r \geqslant 0$ by the suspension isomorphism, we get

$$
H_{i}^{G}\left(X_{r} ; T\right) \cong H_{i-1}^{G}\left(X_{r-1} ; T\right) \text { for all } i \geqslant 2, r \geqslant 1 .
$$

Therefore

$$
H_{i}^{G}\left(X_{r} ; T\right) \cong \begin{cases}H_{i-r}^{G}\left(X_{0} ; T\right) & \text { if } i \geqslant r+1 \\ H_{1}^{G}\left(X_{r-i+1} ; T\right) & \text { if } 1 \leqslant i<r+1 .\end{cases}
$$

The $G$-action on $X_{0}$ is free and $X_{0} / G=\mathbb{R} P^{l-1}$. Hence by Proposition 5.5,

$$
H_{i}^{G}\left(X_{0} ; T\right) \cong \mathbb{Z}_{2} \text { for all } 0 \leqslant i \leqslant l-1 .
$$


Now, considering the long exact sequence of $\left(\Sigma X_{r}, C X_{r}\right)$, we get

$$
H_{i}^{G}\left(\Sigma X_{r}, C X_{r} ; T\right) \cong H_{i}^{G}\left(\Sigma X_{r} ; T\right) \text { for all } i \geqslant 2
$$

and the exact sequence

$$
\begin{aligned}
0 \rightarrow H_{1}^{G}\left(\Sigma X_{r} ; T\right) \rightarrow H_{1}^{G}\left(\Sigma X_{r}, C X_{r} ; T\right) \stackrel{\delta}{\rightarrow} H_{0}^{G}\left(C X_{r} ; T\right) \stackrel{\beta}{\rightarrow} \\
H_{0}^{G}\left(\Sigma X_{r} ; T\right) \rightarrow H_{0}^{G}\left(\Sigma X_{r}, C X_{r} ; T\right) \rightarrow 0 .
\end{aligned}
$$

We also have, by excision, for all $i \geqslant 0$,

$$
H_{i}^{G}\left(\Sigma X_{r}, C X_{r} ; T\right) \cong H_{i}^{G}\left(C X_{r}, X_{r} ; T\right) .
$$

Hence, noting that $\delta$ is the zero map, we obtain by (7),

$$
H_{1}^{G}\left(\Sigma X_{r} ; T\right) \cong \begin{cases}\mathbb{Z}_{2} & \text { if } r=0,1 \\ 0 & \text { if } r \geqslant 2 .\end{cases}
$$

Summarising Equations (7), (8) and (12) we get

\begin{tabular}{|c|c|c|c|}
\hline & $r=0$ & $r=1$ & $r \geqslant 2$ \\
\hline$H_{0}^{G}\left(C X_{r}, X_{r} ; T\right)$ & $\mathbb{Z}_{2}$ & 0 & 0 \\
$H_{1}^{G}\left(C X_{r}, X_{r} ; T\right)$ & $\mathbb{Z}_{2}$ & $\mathbb{Z}_{2}$ & 0 \\
$H_{1}^{G}\left(\Sigma X_{r} ; T\right)$ & $\mathbb{Z}_{2}$ & $\mathbb{Z}_{2}$ & 0 \\
\hline
\end{tabular}

Now, taking into account Equations (6), (9) and (10), it follows from the above table that

$$
H_{i}^{G}\left(D\left(\rho_{l} \oplus \theta_{m}\right), S\left(\rho_{l} \oplus \theta_{m}\right) ; T\right) \cong \begin{cases}\mathbb{Z}_{2} & m \leqslant i \leqslant l+m \\ 0 & \text { otherwise }\end{cases}
$$

\section{Acknowledgements}

We would like to thank the referee for his valuable comments and suggestions which helped us improve the paper.

\section{References}

[1] G.E. Bredon, Equivariant Cohomology Theories, Lecture Notes in Mathematics 34, Springer-Verlag, 1967.

[2] S.R. Costenoble, J.P. May, S. Waner, Equivariant orientation theory, Homology, Homotopy Appl. 3 (2001), 265-339.

[3] S.R. Costenoble, S. Waner, Equivariant Poincaré duality, Michigan Math. J. 39 (1992), 325-351.

[4] S.R. Costenoble, S. Waner, Equivariant simple Poincaré duality, Michigan Math. J. 40 (1993).

[5] M. Datta, N. Pandey, Morse theory on G-manifolds, Topology Appl. 123 (2002), 351-361. 
[6] J.P.C. Greenlees, J.P. May, Equivariant stable homotopy theory, preprint.

[7] J.P. May, A Concise Course in Algebraic Topology, Chicago Lectures in Mathematics Series, University of Chicago Press, Chicago, IL, 1999.

[8] J. Milnor, Morse Theory, Annals of Math. Studies 51, Princeton University Press, Princeton, N.J., 1963.

[9] S. Waner, $G$-CW $(V)$ complexes and $R O(G)$-graded cohomology, in J.P. May, Equivariant homotopy and cohomology theory, CBMS Regional Conference Series in Mathematics 91, American Mathematical Society, Providence, RI, 1996, 89-96.

[10] A.G. Wasserman, Equivariant differential topology, Topology 8 (1969), 127150 .

\author{
Mahuya Datta mahuya@isical.ac.in \\ Statistics and Mathematics Unit \\ Indian Statistical Institute \\ 203, B.T. Road, Kolkata 700108 \\ India
Neeta Pandey pandeyn@gmail.com
Department of Mathematics
Brahmananda Keshab Chandra College
111/2 B.T. Road, Kolkata 700108
India

This article is available at http://intlpress.com/HHA/v9/n1/a19 